\title{
1 LMO2 is critical for early metastatic events in breast cancer
}

\section{Authors:}

3 Shaheen Sikandar ${ }^{1, \dagger, *}$, Jane Antony ${ }^{1, \dagger}$, Gunsagar S. Gulati ${ }^{1, \dagger}$, Angera H. Kuo ${ }^{1}$, William

4 Hai Dang Ho${ }^{1}$, Soumyashree Das ${ }^{2}$, Chloé B. Steen ${ }^{3}$, Thiago Almeida Pereira1, Dalong

5 Qian 1 , Philip A. Beachy ${ }^{1}$, Fredrick Dirbas ${ }^{4}$, Kristy Red-Horse ${ }^{1,2}$, Terence H. Rabbitts ${ }^{5}$,

6 Jean Paul Thiery ${ }^{6}$, Aaron M. Newman ${ }^{1,7, \ddagger}$, and Michael F. Clarke ${ }^{1,8, \ddagger, *}$

\section{Affiliations:}

$8{ }^{1}$ Institute for Stem Cell Biology and Regenerative Medicine, 265 Campus Drive, School of Medicine,

9 Stanford, CA-94305.

102 Department of Biology, Stanford University, Stanford, CA 94305, USA.

$11{ }^{3}$ Division of Oncology, Department of Medicine, Stanford Cancer Institute, Stanford University, Stanford, 12 CA, USA.

$13{ }^{4}$ Department of Surgery, Stanford University School of Medicine, Stanford Cancer Institute, 875 Blake

14 Wilbur Drive, Rm CC2235, Stanford, CA, 94305, USA.

$15{ }^{5}$ Institute of Cancer Research, Division of Cancer Therapeutics, London, SM2 5NG, UK.

$16{ }^{6}$ Bioland Laboratory, Guangzhou Regenerative Medicine and Health, Guangzhou, China.

$17{ }^{7}$ Department of Biomedical Data Science, Stanford University, Stanford, CA 94305, USA.

$18{ }^{8}$ Department of Medicine, Stanford University, Stanford, CA 94305, USA.

19 TThese authors contributed equally.

$20 \quad{ }^{\ddagger}$ Co-senior authors

$21{ }^{*}$ Correspondence to: mfclarke@stanford.edu, ssikanda@ucsc.edu

\section{One sentence summary:}

23 LMO2 modulates STAT3 signaling in breast cancer metastasis. 


\section{SUMMARY}

Metastasis is responsible for the majority of breast cancer-related deaths, however identifying the cellular determinants of metastasis has remained challenging. Here, we identified a minority population of immature $T H Y 1^{+} / V E G F A^{+}$tumor epithelial cells in human breast tumor biopsies that display angiogenic features and are marked by the expression of the oncogene, $\mathrm{LMO} 2$. Higher abundance of $\mathrm{LMO}^{+}$basal cells correlated with tumor endothelial content and predicted poor distant recurrence-free survival in patients. Using MMTV-PYMT/Lmo2 ${ }^{\mathrm{CreERT2}}$ mice, we demonstrated that $L m o 2$ lineagetraced cells have a higher propensity to metastasize. LMO2 knockdown in human breast tumors reduced lung metastasis by impairing intravasation, leading to a reduced frequency of circulating tumor cells. Mechanistically, we find that LMO2 binds to STAT3 and is required for STAT3 activation by TNFa and IL6. Collectively, our study identifies a population of metastasis-initiating cells with angiogenic features and establishes the LMO2-STAT3 signaling axis as a therapeutic target in breast cancer metastasis.

\section{INTRODUCTION}

While significant progress has been made to treat early-stage breast cancer, treatment options and outcomes for metastatic breast cancer have been largely unchanged in a decade (Esposito et al., 2021; Siegel et al., 2011; Siegel et al., 2021). In order to improve outcomes for breast cancer patients, it is critical to identify and elucidate signaling pathways active in metastatic cells. However, it has been difficult to pinpoint cancer cell populations involved in metastasis as they represent a transient state (Lu and Kang, 2019). Previous studies employing lineage tracing and cell surface marker profiling have implicated distinct subsets of tumor epithelial cells in breast cancer metastasis, primarily using lineage markers such as E-cadherin (Beerling et al., 2016, Padmanaban et al., 2019), N-cadherin (Li et al., 2020) and S100a4 (Fischer et al., 2015). Recent studies have also suggested that metastatic cells display hybrid features of both epithelial and mesenchymal lineages (Kröger et al., 2019; Pastushenko et al., 
51 2021). This has led to a debate in the field about the precise molecular identity of

52 metastasis-initiating cells (Lu and Kang, 2019; Shen and Kang, 2019; Ye et al., 2017).

53 Our previous work has demonstrated that in breast cancer, minority populations of

54 phenotypically immature cells in the tumor are enriched in tumor-initiating potential and 55 metastasis (Al-Hajj et al., 2003; Liu et al., 2010; Sikandar et al., 2017). Recent 56 advances in single-cell technologies have revealed complex transcriptional landscapes

57 in human tumors and enabled precise molecular characterization of these minority cell 58 populations (Lawson et al., 2018). However, the functional and clinical significance of 59 these populations remains to be elucidated (Lawson et al., 2018; Tanay and Regev, 60 2017). To understand the transcriptional heterogeneity in breast cancer, we performed 61 single-cell RNA sequencing (scRNA-seq) in primary patient samples and developed a 62 novel computational method that can predict immature cell populations in silico (Gulati 63 et al., 2020). Using our scRNA-seq data, bulk tumor expression deconvolution, lineage 64 tracing, and functional assays, we have now identified a clinically relevant population of 65 metastasis-initiating cells that express the hematopoietic transcription factor and T-cell 66 oncogene, LMO2. Here, we mechanistically define the role of LMO2 in breast cancer 67 metastasis by its association with tumor vasculature and identify LMO2 as a previously unknown regulator of STAT3 signaling in breast cancer.

\section{RESULTS}

LMO2 is expressed in a minority population of immature $\mathrm{THY1}^{+} / \mathrm{VEGFA}{ }^{+}$human breast cancer cells.

72 To understand the substructure of the epithelial populations in breast cancer, we started

73 by analyzing scRNA-seq profiles (Gulati et al., 2020) of human breast tumor epithelial 74 cells from patients with triple-negative $(n=5)$ or estrogen receptor positive $\left(E R^{+}\right)$breast 75 cancer $(n=13)$. We identified a minority population of $\mathrm{THY}^{+}$cells that were largely 76 restricted to the basal compartment, comprising $11 \%$ of all basal cells (Fig. S1A, Table 77 S1). Moreover, within this subset, 33\% of cells expressed VEGFA (Fig. S1A). We were struck by this combination since $\mathrm{THY}^{+}$cells are enriched in reconstitution potential in 
79 the normal mammary gland (Lobo et al., 2018) and tumorigenic potential in mouse

80 tumors (Cho et al., 2008) and VEGFA is a pro-angiogenic factor linked to tumor growth

81 and distant metastasis (Mercurio et al., 2005; Zhao et al., 2015). To determine whether

$82 \mathrm{THY1}^{+} / \mathrm{VEGFA}{ }^{+}$cells represent a potential immature cell population, we applied

83 CytoTRACE, a computational framework for predicting cellular differentiation status on

84 the basis of single-cell transcriptional diversity (Gulati et al., 2020). We found that

85 relative to other basal cells, $\mathrm{THY}^{+} / \mathrm{VEGFA}{ }^{+}$cells are predicted to be significantly less

86 differentiated, suggesting a role for this population in tumor growth or metastasis (Fig.

87 1A).

88 To identify potential molecular regulators within this population, we next searched for genes with expression patterns that overlap THY1 and VEGFA expression in our dataset. Intriguingly, we found that LMO2, a hematopoietic stem cell regulator (Yamada et al., 1998) and T-cell oncogene (Larson, 1995), was among the top five hits (Fig. 1B,

92 Table S2). LMO2 also marked $\mathrm{THY}^{+} / \mathrm{VEGFA}{ }^{+}$cells in an independent scRNA-seq atlas

93 of triple-negative human breast tumors (Kim et al., 2018), corroborating this result (Fig.

94 1C). Analysis of the $\mathrm{LMO}^{+}$basal epithelial subset showed that these cells not only 95 express THY1 and epithelial cytokeratins (Fig. 1D), but also display a coherent gene expression program significantly enriched in angiogenesis genes, including VEGFA and

97 S100A4 (Fig. 1E, Table S3).

We next measured the relative abundance of distinct endothelial, immune, stromal, and epithelial populations in human breast tumors with respect to $\mathrm{LMO}^{+}$basal cells. As LMO2 is expressed in myriad cell types, including immune, stromal, and endothelial cells, the expression of the gene is insufficient to distinguish cell types. Therefore, we defined unique transcriptional signatures for various niche and breast epithelial cells

103 from our scRNA-seq data and utilized CIBERSORTx, a deconvolution approach, to 104 calculate the cellular composition of bulk RNA admixtures from breast cancer clinical 105 cohorts (Newman et al., 2019) (Methods). In line with our previous results, we 106 observed a striking correlation between the abundance of $\mathrm{LMO}^{+}$basal cells and 
107 endothelial cell content imputed in 508 breast tumors (Esserman et al., 2012) $(r=0.45$;

$108 P<2 \times 10^{-16}$; Fig. 1F).

109 Human LMO2 $^{+}$basal cells are associated with poor outcomes in breast cancer 110 patients.

111 Deconvolution of an additional 3,024 human breast tumors from three clinical cohorts

112 (Curtis et al., 2012; TCGA, 2012) revealed that basal LMO2+ cells are more abundant in

113 'Basal' breast cancer subtypes which correlate with more aggressive breast cancers as 114 compared to other PAM50 classes (Perou et al., 2000) (Fig. S1B). We also found a 115 significant increase in basal $\mathrm{LMO}^{+}$cells with worsening clinical grade and stage of the 116 tumor (Fig. S1C, D), suggesting that $\mathrm{LMO}^{+}$cells increase with tumor progression. 117 Importantly, higher levels of $\mathrm{LMO}^{+}$basal cells were significantly associated with inferior 118 distant recurrence-free survival (Fig. 1G), independent of estrogen receptor status. 119 These data link the abundance of $\mathrm{LMO}^{+}$basal epithelial cells with more aggressive 120 breast tumors and distant metastasis.

121 Lmo2 lineage-traced cells have a higher propensity to metastasize.

122 To experimentally verify our in silico findings, we began by employing the CreERT2 123 system (Rios et al., 2014; van Amerongen et al., 2012; Van Keymeulen et al., 2011) to 124 delineate the fate of epithelial cells that have expressed $\mathrm{LMO}^{+}$in breast tumors. We

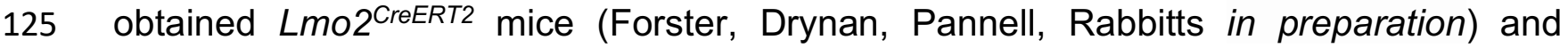
126 crossed them to Rosa $26^{m T m G}$ reporter and MMTV-PyMT tumor mice to generate triple127 transgenic $L m o 2^{\text {CreERT2} / R o s a 26 ~}{ }^{m T m G} / M M T V-P y M T$ mice, which we termed Lmo2-PyMT 128 (Fig. 2A). MMTV-PyMT tumors are an aggressive luminal subtype of breast cancer 129 (Herschkowitz et al., 2007) that metastasize to the lungs (Guy et al., 1992) and have 130 been extensively used to explore the cellular underpinnings of breast cancer metastasis 131 (Beerling et al., 2016; Fischer et al., 2015; Padmanaban et al., 2019; Pastushenko et 132 al., 2018). As Lmo2 is expressed in other cells such as stromal and endothelial cells 133 (Gratzinger et al., 2009), we orthotopically transplanted lineage depleted (CD45-/CD31134 /Ter119-) tumor cells from TdTomato-fluorescent Lmo2-PyMT into non-fluorescent BL6 135 mice to clearly assess the contribution of Lmo2 lineage-traced breast cancer cells from 
136 the tumor. After tumors were formed, we pulsed the mice with tamoxifen to induce 137 expression of GFP in Lmo2-expressing cells (Fig. 2B). At 48h post-pulse, we verified 138 that expression of Lmo2 was enriched in the transplanted GFP ${ }^{+}$cancer cells (Figs. 2C, 139 S2, and S3A). FACS quantification demonstrated that GFP+ cells represented a minor 140 fraction of all tumor cells and expressed the epithelial marker, EpCAM (Fig. 2C).

141 To assess the population dynamics of Lmo2 lineage-traced cells, we plated TdTomato 142 tumor cells from Lmo2-PyMT mice in 3D organoid assays and pulsed the organoids with 143 4-hydroxytamoxifen. Consistent with the in vivo model, lineage-traced $\mathrm{GFP}^{+}$cells 144 comprised a minority of tumor organoids ( $2 \%) 7$ days post-pulse. This percentage was 145 unchanged even after 4 weeks in culture, suggesting similar proliferative capacity 146 between GFP ${ }^{+}$and TdTomato ${ }^{+}$cells (Fig. S3B). We confirmed this by plating sorted 147 GFP $^{+}$and TdTomato $^{+}$cells in 3D organoid cultures and showing that both populations 148 formed organoids at similar frequencies (Fig. S3C).

149 To determine whether $\mathrm{Lmo}^{+}$cells co-associate with endothelial cells, as predicted in 150 silico (Fig. 1F), we stained vasculature with endomucin and visualized their co151 localization with 3D imaging. We found that Lmo2 lineage-traced cells not only resided 152 near tumor blood vessels (Fig. 2E) but surprisingly $\sim 20 \%$ showed co-localization with 153 tumor vasculature and appeared to be incorporated into the tumor vasculature (Fig. 2E 154 and S3D).

155 Given that abundance of $\mathrm{LMO}^{+}$cells in patients predicts distant recurrence-free 156 survival (Fig. 1G) and Lmo2 lineage-traced cells reside closer to tumor vasculature, we 157 next tested whether $\mathrm{Lmo}^{+}$cells have metastatic capabilities. As dissemination of 158 metastatic cells occurs continuously during tumor growth, to lineage-trace tumor cells 159 expressing Lmo2, we pulsed Lmo2-PyMT mice with tamoxifen 2-3 times per week once 160 the tumors were palpable and continued until tumor endpoint (see Methods; Fig. 3F). 161 At the end of the experiment, we found that in the primary tumor only $10-15 \%$ of tumor 162 cells were GFP $^{+}$(Fig. 2G). Surprisingly, even though the tumor was majority 163 TdTomato $^{+}$, the lungs had a disproportionately higher number of $\mathrm{GFP}^{+}$metastases, 
several of which were also larger than the TdTomato ${ }^{+}$metastases $(P=0.034$, Wilcoxon signed-rank unpaired test) (Fig. $2 \mathrm{H}$ ). These data suggest that $L m o 2$ lineage-traced cells have a higher propensity to form metastases in the PyMT mice and is consistent with our findings in human breast cancer patients (Fig. 1G). Furthermore, a subset of GFP tumor cells did not remain Lmo2 positive (Fig. S3E), suggesting that expression of Lmo2 in some cells represents a transient state, in agreement with previous studies linking transient cell states to metastases (Pastushenko et al., 2018).

\section{LMO2 knockdown abrogates lung metastasis in human breast cancer models.}

To understand the functional role of LMO2 in human breast cancer, we knocked down LMO2 expression in MDA-MB-468 cells using two independent shRNA vectors tagged with a GFP reporter (Fig. S4A-C). We then implanted the cells orthotopically in immunodeficient mice (Fig. 3A and S4D). In contrast to a previous report (Liu et al., 2016), knockdown of $L M O 2$ did not affect primary tumor growth (Fig. 3B) or proliferation in vitro (Fig. S5A). Nevertheless, LMO2-knockdown tumors had significantly fewer lung metastases relative to control ( $P=0.003$, ANOVA; Fig. 2C). Moreover, LMO2knockdown in tumor-bearing mice led to a significantly reduced number of circulating tumor cells compared to control mice ( $P<0.0001$, ANOVA; Fig. 2D), implicating LMO2 in tumor cell shedding, a key step in metastasis initiation. To extend our findings to more clinically relevant models, we used patient-derived xenograft (PDX) models previously generated in our lab (Sikandar et al., 2017). Consistent with our MDA-MB468 studies, knockdown of LMO2 dramatically decreased metastasis to the lung in three different PDX models of breast cancer (Fig. 3E, F), but did not significantly impact tumor growth (Fig. S5B-D).

To better understand how LMO2 affects metastasis, we rigorously studied the effects of LMO2 knockdown in vitro in MDA-MB-468 cells. Knockdown of LMO2 showed significant impairment in the ability of cancer cells to migrate across transwells and invade through a 3D hydrogel matrix (Fig. S6A, B). Importantly, since $\mathrm{LMO}^{+}$epithelial cells associated with endothelial cells in patient samples, we tested whether knockdown of $L M O 2$ decreased this association in co-culture assays. We found that in $3 \mathrm{D}$ co- 
193 culture assays with human vascular endothelial cells (HUVECs), LMO2 knockdown 194 significantly impacted incorporation of cancer cells into HUVEC tubes (Fig. S6C). To confirm that the effects of knockdown were specific to LMO2, we overexpressed LMO2

196 in cells with shRNA targeting the 3'UTR. We found that all phenotypes of migration (Fig. 197 3G), invasion (Fig. 3H), and incorporation into the vasculature in vitro (Fig. 3I) could be 198 rescued by overexpression of LMO2 in LMO2-deficient cells. Lastly, to test whether 199 LMO2 is required after metastatic cells enter circulation, we injected control and LMO2 200 knockdown cells into the tail vein. We found that LMO2 knockdown did not significantly 201 impact the formation of lung metastases when cells were directly injected in the tail vein, 202 suggesting that LMO2 is critical for the initial dissemination of cancer cells from the 203 tumor, but not extravasation and formation of metastatic foci (Fig. S6D).

RNA sequencing identifies LMO2 as a regulator of IL6-JAK-STAT3 signaling.

To elucidate the molecular function of LMO2 in breast cancer cells, we performed bulk RNA sequencing of MDA-MB-468 cells after transfection with control and LMO2 shRNA vectors (Fig. 4A). Among the top 50 genes downregulated after LMO2 knockdown were genes previously implicated in metastasis, such as BMP2 (Bach et al., 2018; Huang et al., 2017; Wang et al., 2017), LGR6 (Leushacke and Barker, 2012; Ruan et al., 2019), EGR4 (Matsuo et al., 2014), TDO2 (D'Amato et al., 2015) and S100A4 (Boye and

211 Maelandsmo, 2010; Garrett et al., 2006; Helfman et al., 2005) (Fig. 4A, Table S4). 212 Using Gene Set Enrichment Analysis (GSEA) (Mootha et al., 2003; Subramanian et al., 213 2005), we found that inflammatory pathways, such as TNF $\alpha$ via NF-kB signaling, IL6214 JAK-STAT3 signaling, and IFN $\gamma$ response, were significantly downregulated in LMO2 215 knockdown as compared to control conditions (Fig. 4B). To confirm our findings in 216 primary patient samples we performed single-sample GSEA in our scRNA-seq data set 217 as well as a larger published dataset of primary human breast cancer cells (Kim et al., 218 2018). We found that IL6-JAK-STAT3 signaling was significantly enriched in LMO2 $^{+}$ 219 versus LMO2$^{-}$single cells (Fig. 4C) compared to other pathways (Fig. S7). In the 220 hematopoietic system, LMO2 is an adaptor protein that facilitates formation of functional 221 protein complexes which then activate transcription of downstream targets (Chambers 222 and Rabbitts, 2015). Hence, we asked whether LMO2 may similarly behave as a 
223 bridging molecule to drive downstream signaling in breast epithelial cells. Using 224 proximity ligation assays, we found that LMO2 had a significantly high binding affinity to 225 STAT3, but not to NF-kB, further confirming our pathway analysis (Fig. 4D).

226 LMO2 is required for STAT3 activation by IL6 and TNF $\alpha$.

227 To demonstrate specificity and functional significance of the LMO2-STAT3 interaction, 228 we first showed that LMO2 knockdown significantly reduced LMO2-STAT3 binding $(P<$ 229 0.0001, ANOVA; Fig. 5A). We also confirmed the LMO2-STAT3 interaction using co230 immunoprecipitation assays (Co-IP) of LMO2 with STAT3 (Fig. 5B) and, a reverse Co231 IP of STAT3 with LMO2 (Fig. 5C). In breast cancer, STAT3 is activated by cytokines, 232 such as IL6 (Zhong et al., 1994), TNFa (De Simone et al., 2015), IFNa (Beadling et al., 233 1994; Cho et al., 1996; Darnell et al., 1994) and IFN $\gamma$ (Darnell et al., 1994; Will et al., 234 1996), as well as receptor tyrosine kinases such as EGFR (Kim et al., 2012; Zhao et al., 235 2020), leading to phosphorylation of STAT3. Dimerization of pSTAT3 and translocation 236 to the nucleus activates transcription of downstream target genes involved in several 237 processes, including metastasis. To understand whether the STAT3-LMO2 interaction 238 has an effect on downstream STAT3 signaling, we used a STAT3-luciferase reporter 239 assay. We stimulated control or LMO2 knockdown cells with IL6, TNFa, IFN $\gamma$, IFNa, and 240 EGF. We found that cells with knockdown of LMO2 were unable to induce transcription 241 of the STAT3-luciferase reporter when treated with IL6 and TNFa as compared to 242 control (Fig. 5D), but STAT3-luciferase was activated by IFN $\gamma$, IFNa, and EGFR 243 treatment. This suggests that LMO2 function in breast cancer cells is specific to 244 activation of STAT3 signaling through IL6 and TNFa. On a molecular level, we found 245 that knockdown of LMO2 significantly reduced STAT3 phosphorylation at Tyr705, which 246 is required for its dimerization and transcriptional activity (Fig. 5E and Fig. S8). To 247 understand how LMO2 regulates phosphorylation of STAT3, we examined the 248 interaction of STAT3 with its upstream activator JAK2 and its cytoplasmic inhibitor 249 PIAS3. Knockdown of LMO2 decreased the interaction of STAT3 with JAK2 (Fig. 5F) 250 and allowed for increased interaction with its inhibitor, PIAS3 (Fig. 5G). This suggests 251 that LMO2 works as an adaptor protein in the cytoplasm to stabilize the STAT3-JAK2 252 interaction, thereby allowing efficient phosphorylation and activation of STAT3 while 
253 simultaneously preventing its negative regulation by PIAS3 (Fig. 5H). This LMO2-

254 mediated control of a core inflammatory response pathway could enable cancer cells to 255 rapidly transition between cellular phenotypes required for metastasis and represents a 256 therapeutic vulnerability that could be targeted.

\section{DISCUSSION}

258 Efficient metastasis of tumor cells requires transition from a proliferative to an invasive 259 state and back to a proliferative state at a distant site (Beerling et al., 2016). Previous 260 studies using mouse tumor models have demonstrated the requirement of a basal 261 epithelial program in metastasis (Cheung et al., 2013; Padmanaban et al., 2019) and 262 showed that hybrid epithelial-mesenchymal states (Beerling et al., 2016; Kröger et al., 263 2019; Nieto et al., 2016) in metastasis express angiogenic factors (Pastushenko et al., 264 2018). Here, we have identified a population of $\mathrm{THY}^{+} / \mathrm{VEGFA}{ }^{+}$human basal epithelial 265 cells with higher transcriptional diversity that is marked by transient expression of 266 LMO2. Moreover, we demonstrate that Lmo2 lineage-traced epithelial cells have a higher propensity to form lung metastases. Moreover, knockdown of LMO2 decreases lung metastasis in multiple tumor models of human breast cancer by affecting multiple steps during intravasation. It is important to note that only a subset of Lmo2 lineage-

271 activated in the presence of TNF $\alpha$ and IL6 from the microenvironment. Our observations 272 highlight a heterogenous, cancer-cell-intrinsic response to the microenvironment while 273 previous studies have demonstrated that there is a reciprocal effect of cancer cells on 274 the tumor microenvironment with recruitment of macrophages and cross-talk with tumor 275 endothelial cells during metastasis (Borriello et al., 2020).

276 LMO2 has been extensively studied in hematological malignancies and is well277 established as a transcriptional adaptor protein (Chambers and Rabbitts, 2015). Recent 278 studies have attempted to understand the role of LMO2 in breast cancer (Hu et al., 279 2021; Liu et al., 2016; Liu et al., 2017) but have suffered from contradictory results, 280 were limited to cell lines, and did not attribute LMO2 to any particular tumor cell 281 population. We demonstrate that LMO2 is a previously unidentified binding partner of 
282 STAT3 in breast cancer cells and modulates STAT3 signaling in response to IL6 and

283 TNFa. We speculate that the expression of LMO2 provides the necessary threshold to 284 stabilize STAT3 signaling, which in turn enables the tumor cells to enter a transient 285 metastatic state (Wendt et al., 2014) and escape the primary tumor. STAT3 signaling is 286 involved in a number of processes and its targets may be defined in unison with other 287 contextual signals such as inflammation. Several studies have linked low chronic 288 inflammation in cancer to metastasis (Joyce and Pollard, 2009; Liu et al., 2015). We 289 speculate that LMO2 is a critical molecular link between these processes and define a 290 novel function for LMO2 in breast cancer metastasis. The development of new methods 291 targeting adaptor proteins (Wang et al., 2020) and small molecules that disrupt the 292 LMO2-STAT3 axis (Milton-Harris et al., 2020) could provide novel therapeutic strategies 293 to modulate STAT3 signaling and inhibit metastatic colonization in breast cancer. 


\section{REFERENCES AND NOTES}

295

Al-Hajj, M., Wicha, M.S., Benito-Hernandez, A., Morrison, S.J., and Clarke, M.F. (2003). Prospective identification of tumorigenic breast cancer cells. Proc Natl Acad Sci U S A 100, 3983-3988.

Bach, D.H., Park, H.J., and Lee, S.K. (2018). The Dual Role of Bone Morphogenetic Proteins in Cancer. Mol Ther Oncolytics 8, 1-13.

Beadling, C., Guschin, D., Witthuhn, B.A., Ziemiecki, A., Ihle, J.N., Kerr, I.M., and Cantrell, D.A. (1994). Activation of JAK kinases and STAT proteins by interleukin-2 and interferon alpha, but not the $\mathrm{T}$ cell antigen receptor, in human $\mathrm{T}$ lymphocytes. EMBO J 13, 5605-5615.

Beerling, E., Seinstra, D., de Wit, E., Kester, L., van der Velden, D., Maynard, C., Schäfer, R., van Diest, P., Voest, E., van Oudenaarden, A., et al. (2016). Plasticity between Epithelial and Mesenchymal States Unlinks EMT from Metastasis-Enhancing Stem Cell Capacity. Cell Reports 14, 2281-2288.

Borriello, L., Karagiannis, G.S., Duran, C.L., Coste, A., Oktay, M.H., Entenberg, D., and Condeelis, J.S. (2020). The role of the tumor microenvironment in tumor cell intravasation and dissemination. European Journal of Cell Biology 99, 151098. Boye, K., and Maelandsmo, G.M. (2010). S100A4 and metastasis: a small actor playing many roles. Am J Pathol 176, 528-535.

Chambers, J., and Rabbitts, T.H. (2015). LMO2 at 25 years: a paradigm of chromosomal translocation proteins. Open Biol 5, $150062-150062$.

Cheung, K.J., Gabrielson, E., Werb, Z., and Ewald, A.J. (2013). Collective invasion in breast cancer requires a conserved basal epithelial program. Cell 155, 1639-1651. Cho, R.W., Wang, X., Diehn, M., Shedden, K., Chen, G.Y., Sherlock, G., Gurney, A., Lewicki, J., and Clarke, M.F. (2008). Isolation and Molecular Characterization of Cancer Stem Cells in MMTV-Wnt-1 Murine Breast Tumors. STEM CELLS 26, 364-371. Cho, S.S., Bacon, C.M., Sudarshan, C., Rees, R.C., Finbloom, D., Pine, R., and O'Shea, J.J. (1996). Activation of STAT4 by IL-12 and IFN-alpha: evidence for the involvement of ligand-induced tyrosine and serine phosphorylation. J Immunol 157, 4781-4789.

Curtis, C., Shah, S.P., Chin, S.F., Turashvili, G., Rueda, O.M., Dunning, M.J., Speed, D., Lynch, A.G., Samarajiwa, S., Yuan, Y., et al. (2012). The genomic and transcriptomic architecture of 2,000 breast tumours reveals novel subgroups. Nature 486, 346-352.

D'Amato, N.C., Rogers, T.J., Gordon, M.A., Greene, L.I., Cochrane, D.R., Spoelstra, N.S., Nemkov, T.G., D'Alessandro, A., Hansen, K.C., and Richer, J.K. (2015). A TDO2AhR signaling axis facilitates anoikis resistance and metastasis in triple-negative breast cancer. Cancer Res 75, 4651-4664.

Darnell, J.E., Jr., Kerr, I.M., and Stark, G.R. (1994). Jak-STAT pathways and transcriptional activation in response to IFNs and other extracellular signaling proteins. Science 264, 1415-1421.

De Simone, V., Franze, E., Ronchetti, G., Colantoni, A., Fantini, M.C., Di Fusco, D., Sica, G.S., Sileri, P., MacDonald, T.T., Pallone, F., et al. (2015). Th17-type cytokines, IL-6 and TNF-alpha synergistically activate STAT3 and NF-kB to promote colorectal cancer cell growth. Oncogene 34, 3493-3503. 
Esposito, M., Ganesan, S., and Kang, Y. (2021). Emerging strategies for treating metastasis. Nature Cancer 2, 258-270.

Esserman, L.J., Berry, D.A., Cheang, M.C., Yau, C., Perou, C.M., Carey, L., DeMichele, A., Gray, J.W., Conway-Dorsey, K., Lenburg, M.E., et al. (2012). Chemotherapy response and recurrence-free survival in neoadjuvant breast cancer depends on biomarker profiles: results from the I-SPY 1 TRIAL (CALGB 150007/150012; ACRIN 6657). Breast Cancer Res Treat 132, 1049-1062.

Fischer, K.R., Durrans, A., Lee, S., Sheng, J., Li, F., and Wong, S.T. (2015). Epithelialto-mesenchymal transition is not required for lung metastasis but contributes to chemoresistance. Nature 527.

Garrett, S.C., Varney, K.M., Weber, D.J., and Bresnick, A.R. (2006). S100A4, a mediator of metastasis. J Biol Chem 281, 677-680.

Gratzinger, D., Zhao, S., West, R., Rouse, R.V., Vogel, H., Gil, E.C., Levy, R., Lossos, I.S., and Natkunam, Y. (2009). The transcription factor LMO2 is a robust marker of vascular endothelium and vascular neoplasms and selected other entities. Am J Clin Pathol 131, 264-278.

Gulati, G.S., Sikandar, S.S., Wesche, D.J., Manjunath, A., Bharadwaj, A., Berger, M.J., llagan, F., Kuo, Angera H., Hsieh, R.W., Cai, S., et al. (2020). Single-cell transcriptional diversity is a hallmark of developmental potential. Science 367, 405-411.

Guy, C.T., Cardiff, R.D., and Muller, W.J. (1992). Induction of mammary tumors by expression of polyomavirus middle $T$ oncogene: a transgenic mouse model for metastatic disease. Molecular and Cellular Biology 12, 954-961. Helfman, D.M., Kim, E.J., Lukanidin, E., and Grigorian, M. (2005). The metastasis associated protein S100A4: role in tumour progression and metastasis. Br J Cancer 92, 1955-1958.

Herschkowitz, J.I., Simin, K., Weigman, V.J., Mikaelian, I., Usary, J., Hu, Z., Rasmussen, K.E., Jones, L.P., Assefnia, S., Chandrasekharan, S., et al. (2007). Identification of conserved gene expression features between murine mammary carcinoma models and human breast tumors. Genome Biology 8, R76. Hu, A., Hong, F., Li, D., Xie, Q., Chen, K., Zhu, L., and He, H. (2021). KDM3B-ETF1 fusion gene downregulates LMO2 via the WNT/ $\beta$-catenin signaling pathway, promoting metastasis of invasive ductal carcinoma. Cancer Gene Therapy. Huang, P., Chen, A., He, W., Li, Z., Zhang, G., Liu, Z., Liu, G., Liu, X., He, S., Xiao, G., et al. (2017). BMP-2 induces EMT and breast cancer stemness through Rb and CD44. Cell Death Discov 3, 17039.

Joyce, J.A., and Pollard, J.W. (2009). Microenvironmental regulation of metastasis. Nature reviews Cancer 9, 239-252.

Kim, C., Gao, R., Sei, E., Brandt, R., Hartman, J., Hatschek, T., Crosetto, N., Foukakis, T., and Navin, N.E. (2018). Chemoresistance Evolution in Triple-Negative Breast Cancer Delineated by Single-Cell Sequencing. Cell 173, 879-893.e813.

Kim, S.M., Kwon, O.J., Hong, Y.K., Kim, J.H., Solca, F., Ha, S.J., Soo, R.A., Christensen, J.G., Lee, J.H., and Cho, B.C. (2012). Activation of IL-6R/JAK1/STAT3 signaling induces de novo resistance to irreversible EGFR inhibitors in non-small cell lung cancer with T790M resistance mutation. Mol Cancer Ther 11, 2254-2264. Kröger, C., Afeyan, A., Mraz, J., Eaton, E.N., Reinhardt, F., Khodor, Y.L., Thiru, P., Bierie, B., Ye, X., Burge, C.B., et al. (2019). Acquisition of a hybrid E/M state is 
essential for tumorigenicity of basal breast cancer cells. Proceedings of the National Academy of Sciences of the United States of America 116, 7353-7362.

Larson, R.C., Osada, H., Larson, T.A., Lavenir, I. \& Rabbitts, T.H (1995). The oncogenic LIM protein Rbtn2 causes thymic developmental aberrations that precede malignancy in transgenic mice. Oncogene 11, 853-862.

390 Lawson, D.A., Kessenbrock, K., Davis, R.T., Pervolarakis, N., and Werb, Z. (2018). Tumour heterogeneity and metastasis at single-cell resolution. Nat Cell Biol 20,1349-

3921360.

393 Leushacke, M., and Barker, N. (2012). Lgr5 and Lgr6 as markers to study adult stem cell roles in self-renewal and cancer. Oncogene 31, 3009-3022. Li, Y., Lv, Z., Zhang, S., Wang, Z., He, L., Tang, M., Pu, W., Zhao, H., Zhang, Z., Shi, Q., et al. (2020). Genetic Fate Mapping of Transient Cell Fate Reveals N-Cadherin Activity and Function in Tumor Metastasis. Dev Cell 54, 593-607.e595. Liu, H., Patel, M.R., Prescher, J.A., Patsialou, A., Qian, D., Lin, J., Wen, S., Chang, Y.F., Bachmann, M.H., Shimono, Y., et al. (2010). Cancer stem cells from human breast tumors are involved in spontaneous metastases in orthotopic mouse models. Proceedings of the National Academy of Sciences 107, 18115-18120. Liu, J., Lin, P.C., and Zhou, B.P. (2015). Inflammation fuels tumor progress and metastasis. Curr Pharm Des 21, 3032-3040. Sun, W. (2016). LMO2 attenuates tumor growth by targeting the Wnt signaling pathway in breast and colorectal cancer. Sci Rep 6, 36050-36050. Liu, Y., Wang, Z., Huang, D., Wu, C., Li, H., Zhang, X., Meng, B., Li, Z., Zhu, T., Yang, S., et al. (2017). LMO2 promotes tumor cell invasion and metastasis in basal-type breast cancer by altering actin cytoskeleton remodeling. Oncotarget 8, 9513-9524. Lobo, N.A., Zabala, M., Qian, D., and Clarke, M.F. (2018). Serially transplantable mammary epithelial cells express the Thy-1 antigen. Breast Cancer Res 20,121-121. Lu, W., and Kang, Y. (2019). Epithelial-Mesenchymal Plasticity in Cancer Progression and Metastasis. Dev Cell 49, 361-374. Matsuo, T., Dat le, T., Komatsu, M., Yoshimaru, T., Daizumoto, K., Sone, S., Nishioka, Y., and Katagiri, T. (2014). Early growth response 4 is involved in cell proliferation of small cell lung cancer through transcriptional activation of its downstream genes. PloS one 9 , e113606.

Mercurio, A.M., Lipscomb, E.A., and Bachelder, R.E. (2005). Non-Angiogenic Functions of VEGF in Breast Cancer. Journal of Mammary Gland Biology and Neoplasia 10, 283290.

Milton-Harris, L., Jeeves, M., Walker, S.A., Ward, S.E., and Mancini, E.J. (2020). Small molecule inhibits T-cell acute lymphoblastic leukaemia oncogenic interaction through conformational modulation of LMO2. Oncotarget; Vol 11, No 19. Mootha, V.K., Lindgren, C.M., Eriksson, K.F., Subramanian, A., Sihag, S., Lehar, J., Puigserver, P., Carlsson, E., Ridderstrale, M., Laurila, E., et al. (2003). PGC-1alpharesponsive genes involved in oxidative phosphorylation are coordinately downregulated in human diabetes. Nat Genet 34, 267-273.

428 Newman, A.M., Steen, C.B., Liu, C.L., Gentles, A.J., Chaudhuri, A.A., Scherer, F., 429 Khodadoust, M.S., Esfahani, M.S., Luca, B.A., Steiner, D., et al. (2019). Determining 
cell type abundance and expression from bulk tissues with digital cytometry. Nature Biotechnology 37, 773-782.

Nieto, M.A., Huang, R.Y., Jackson, R.A., and Thiery, J.P. (2016). Emt: 2016. Cell 166, 21-45.

Padmanaban, V., Krol, I., Suhail, Y., Szczerba, B.M., Aceto, N., Bader, J.S., and Ewald, A.J. (2019). E-cadherin is required for metastasis in multiple models of breast cancer. Nature 573, 439-444. Pastushenko, I., Brisebarre, A., Sifrim, A., Fioramonti, M., Revenco, T., Boumahdi, S., Van Keymeulen, A., Brown, D., Moers, V., Lemaire, S., et al. (2018). Identification of the tumour transition states occurring during EMT. Nature 556, 463-468. Pastushenko, I., Mauri, F., Song, Y., de Cock, F., Meeusen, B., Swedlund, B., Impens, F., Van Haver, D., Opitz, M., Thery, M., et al. (2021). Fat1 deletion promotes hybrid EMT state, tumour stemness and metastasis. Nature 589, 448-455. Perou, C.M., Sørlie, T., Eisen, M.B., van de Rijn, M., Jeffrey, S.S., Rees, C.A., Pollack, J.R., Ross, D.T., Johnsen, H., Akslen, L.A., et al. (2000). Molecular portraits of human breast tumours. Nature 406, 747-752. Rios, A.C., Fu, N.Y., Lindeman, G.J., and Visvader, J.E. (2014). In situ identification of bipotent stem cells in the mammary gland. Nature 506, 322-327. Ruan, X., Liu, A., Zhong, M., Wei, J., Zhang, W., Rong, Y., Liu, W., Li, M., Qing, X., Chen, G., et al. (2019). Silencing LGR6 Attenuates Stemness and Chemoresistance via Inhibiting Wnt/ $\beta-$ Catenin Signaling in Ovarian Cancer. Molecular therapy oncolytics 14, 94-106. Shen, M., and Kang, Y. (2019). Role Reversal: A Pro-metastatic Function of ECadherin. Dev Cell 51, 417-419. Cancer Journal for Clinicians 61, 212-236. Siegel, R.L., Miller, K.D., Fuchs, H.E., and Jemal, A. (2021). Cancer Statistics, 2021. CA: A Cancer Journal for Clinicians 71, 7-33.

Sikandar, S.S., Kuo, A.H., Kalisky, T., Cai, S., Zabala, M., Hsieh, R.W., Lobo, N.A., Scheeren, F.A., Sim, S., Qian, D., et al. (2017). Role of epithelial to mesenchymal transition associated genes in mammary gland regeneration and breast tumorigenesis. Nature Communications 8, 1669.

Subramanian, A., Tamayo, P., Mootha, V.K., Mukherjee, S., Ebert, B.L., Gillette, M.A., Paulovich, A., Pomeroy, S.L., Golub, T.R., Lander, E.S., et al. (2005). Gene set enrichment analysis: a knowledge-based approach for interpreting genome-wide expression profiles. Proc Natl Acad Sci U S A 102, 15545-15550.

475

Tanay, A., and Regev, A. (2017). Scaling single-cell genomics from phenomenology to mechanism. Nature 541, 331-338.

TCGA (2012). Comprehensive molecular portraits of human breast tumours. Nature 490, 61-70.

van Amerongen, R., Bowman, A.N., and Nusse, R. (2012). Developmental stage and time dictate the fate of Wnt/beta-catenin-responsive stem cells in the mammary gland. Cell Stem Cell 11, 387-400.

Van Keymeulen, A., Rocha, A.S., Ousset, M., Beck, B., Bouvencourt, G., Rock, J., Sharma, N., Dekoninck, S., and Blanpain, C. (2011). Distinct stem cells contribute to mammary gland development and maintenance. Nature 479, 189-193. 
476 Wang, M.H., Zhou, X.M., Zhang, M.Y., Shi, L., Xiao, R.W., Zeng, L.S., Yang, X.Z.,

477 Zheng, X.F.S., Wang, H.Y., and Mai, S.J. (2017). BMP2 promotes proliferation and

478 invasion of nasopharyngeal carcinoma cells via mTORC1 pathway. Aging (Albany NY)

479 9, 1326-1340.

480 Wang, Y., Jiang, X., Feng, F., Liu, W., and Sun, H. (2020). Degradation of proteins by

481 PROTACs and other strategies. Acta Pharm Sin B 10, 207-238.

482 Wendt, M.K., Balanis, N., Carlin, C.R., and Schiemann, W.P. (2014). STAT3 and

483 epithelial-mesenchymal transitions in carcinomas. JAKSTAT 3, e28975.

484 Will, A., Hemmann, U., Horn, F., Rollinghoff, M., and Gessner, A. (1996). Intracellular

485 murine IFN-gamma mediates virus resistance, expression of oligoadenylate synthetase, 486 and activation of STAT transcription factors. J Immunol 157, 4576-4583.

487 Yamada, Y., Warren, A.J., Dobson, C., Forster, A., Pannell, R., and Rabbitts, T.H.

488 (1998). The T cell leukemia LIM protein Lmo2 is necessary for adult mouse

489 hematopoiesis. Proceedings of the National Academy of Sciences of the United States

490 of America 95, 3890-3895.

491 Ye, X., Brabletz, T., Kang, Y., Longmore, G.D., Nieto, M.A., Stanger, B.Z., Yang, J., and

492 Weinberg, R.A. (2017). Upholding a role for EMT in breast cancer metastasis. Nature

493 547, E1-E3.

494 Zhao, C., Yang, L., Zhou, F., Yu, Y., Du, X., Xiang, Y., Li, C., Huang, X., Xie, C., Liu, Z.,

495 et al. (2020). Feedback activation of EGFR is the main cause for STAT3 inhibition-

496 irresponsiveness in pancreatic cancer cells. Oncogene 39, 3997-4013.

497 Zhao, D., Pan, C., Sun, J., Gilbert, C., Drews-Elger, K., Azzam, D.J., Picon-Ruiz, M.,

498 Kim, M., Ullmer, W., El-Ashry, D., et al. (2015). VEGF drives cancer-initiating stem cells

499 through VEGFR-2/Stat3 signaling to upregulate Myc and Sox2. Oncogene 34, 3107-

5003119.

501 Zhong, Z., Wen, Z., and Darnell, J.E., Jr. (1994). Stat3: a STAT family member

502 activated by tyrosine phosphorylation in response to epidermal growth factor and

503 interleukin-6. Science 264, 95-98. 


\section{ACKNOWLEDGMENTS}

505

506

507

508

509

510

511

512

513

514

515

516

517

518

519

520

521

522

523

524

525

526

527

528

529

530

531

532

533

\section{Supplementary Materials:}

535 Materials and Methods

536 Figures S1-S8

537 Tables S1-S5 grant NS069375.

\section{AUTHOR CONTRIBUTIONS} manuscript.

We thank Patricia Lovelace, Catherine Carswell Crumpton, and other flow cytometry staff for their help and animal facility core members. The Wolverine Aria instrument was funded by NHI grant S10-1S10RR02933801. We thank Diane Heiser for technical assistance with the tail vein injections. We thank Margaret Cuadro for administrative assistance. We thank the Stanford Neuroscience Microscopy Service, supported by NIH

Funding: This work was supported by NIH/NCI (U01CA154209-01 and P01 CA13949005), the Breast Cancer Research Foundation (to M.F.C.), the U.S. Department of Defense (W81XWH-11-1-0287 and W81XWH-13-1-0281 to M.F.C.; S.S.S., W81XWH12-1-0020), the National Cancer Institute (A.M.N., R00CA187192-03; M.F.C., 5R01CA100225-09; G.S.G., PHS grant no. CA09302), the Stanford Bio-X Interdisciplinary Initiatives Seed Grants Program (IIP) (A.M.N., M.F.C.), the Virginia and D.K. Ludwig Fund for Cancer Research (A.M.N., M.F.C), the Stinehart-Reed Foundation (A.M.N.), the Stanford School of Medicine Dean's Fellowship (J.A.), Stanford Bio-X Bowes Graduate Student Fellowship (G.S.G.), and the Stanford Medical Science Training Program (G.S.G.). K.R-H. is a New York Stem Cell Foundation - Robertson Investigator. K.R-H. is also supported by the NIH (R01-HL128503)

S.S.S. and M.F.C. conceived and designed the study. S.S.S. and J.A. performed experiments and analyzed data with supervision from M.F.C. G.S.G. analyzed singlecell and bulk RNA sequencing data with assistance from C.B.S. and supervision from A.M.N. A.H.K. assisted with the PDX studies. W.H.D.H. assisted with the metastasis experiments. S.D. performed staining for visualization of tumor vasculature under the supervision of K.R-H. T.A.P. assisted with the circulating cells experiment under the supervision of P.B. D.Q. provided technical support. F.D. assisted with the collection of patient specimens. J.P.T. assessed the enrichment of genes in $\mathrm{LMO}^{+}$cells and provided guidance in the project. T.R provided the Lmo2 ${ }^{C r E E R T 2}$ mice. S.S.S., J.A., G.S.G., A.M.N. and M.F.C., wrote the manuscript. All authors commented on the 


\section{Figures and Figure Legends}

A

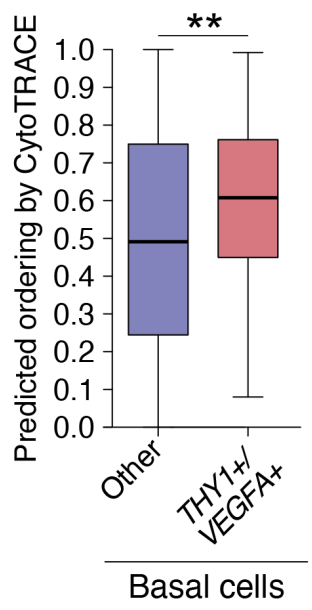

B

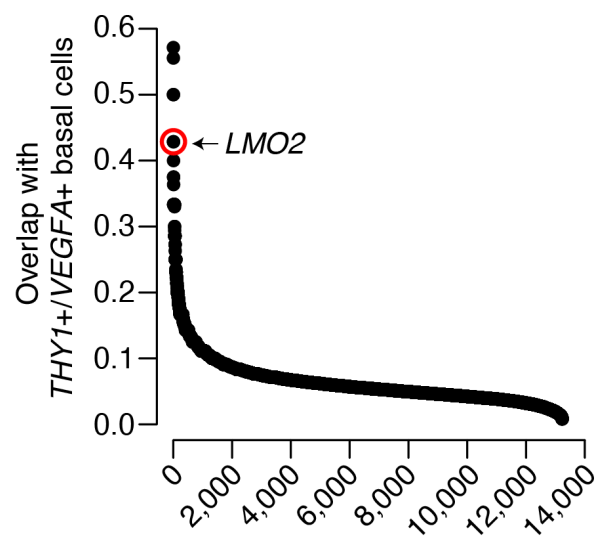

Protein-coding genes expressed by $>5$ basal cells $(n=13,230)$

C

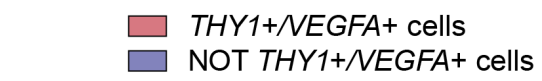

Fisher's combined $P$ value: $2.1 \times 10^{-4}$

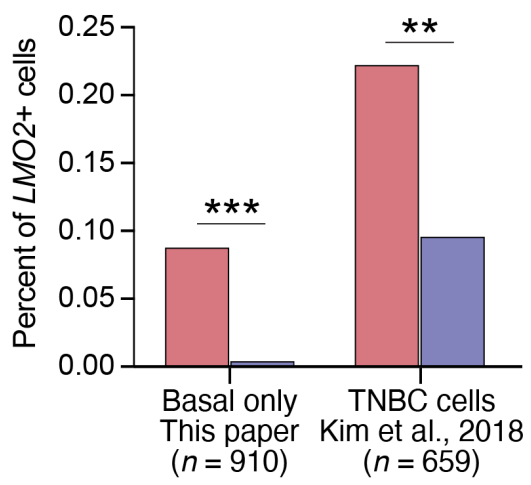

Human breast cancer datasets
D

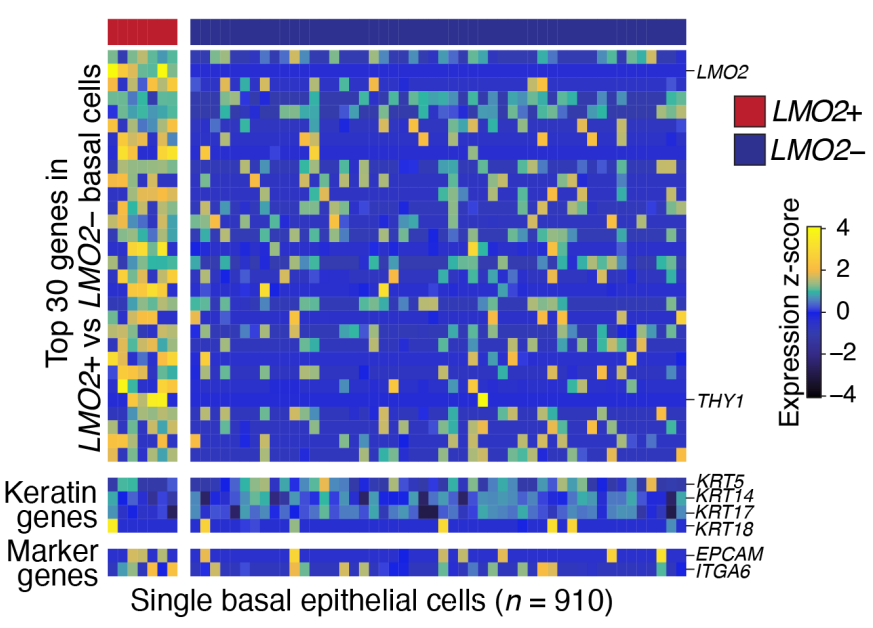

$\mathbf{F}$

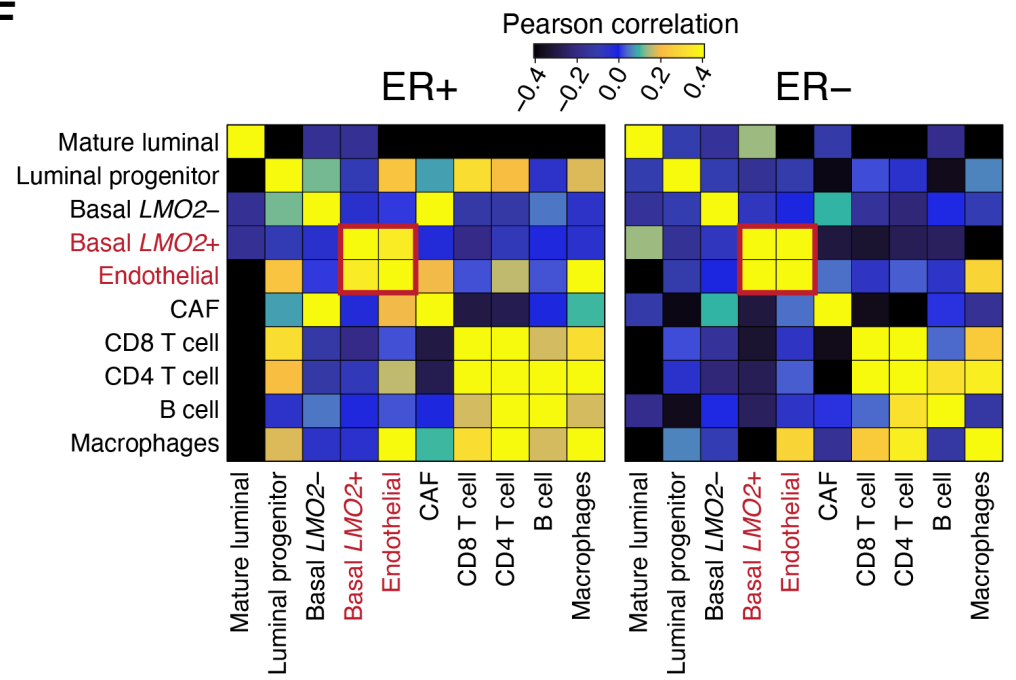

E

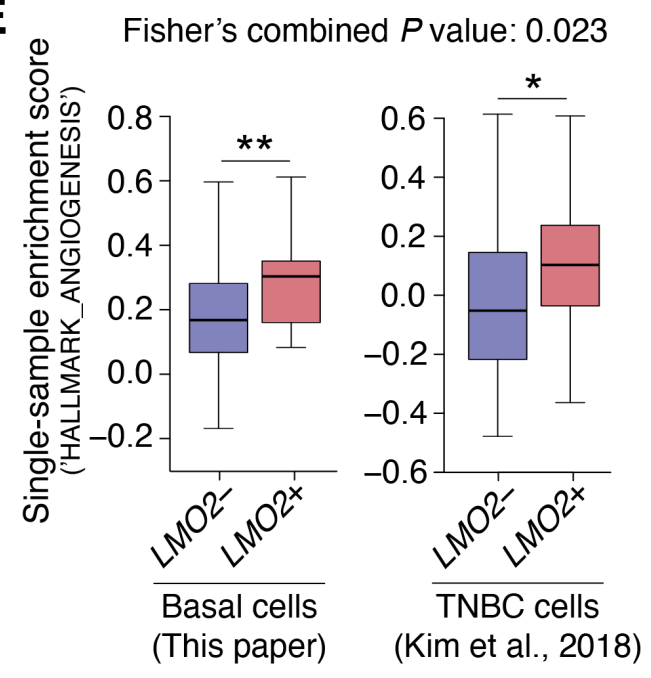

G

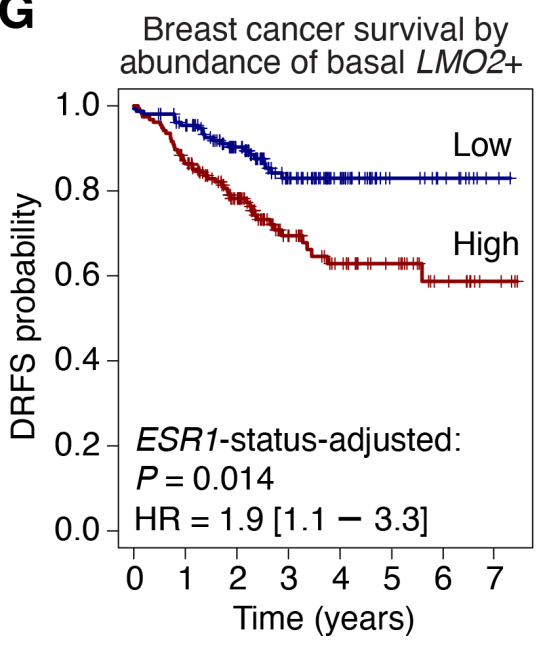


Figure 1: Identification of an immature basal epithelial population associated with pro-angiogenic signaling and poor survival in human breast cancer.

540 (A) Differentiation scores of basal epithelial cells from 17 human breast tumors profiled by scRNA-seq (all but 'SU196' contained basal cells). Differentiation scores were determined by CytoTRACE (Gulati et al., 2020). Statistical significance between $\mathrm{THY}^{+} /$ VEGFA $A^{+}$basal cells and other basal cells was calculated using an unpaired two-tailed $t$ test. ${ }^{*} P<0.1 ;{ }^{* *} P<0.05 ;{ }^{* *} P<0.01$. (B) Plot showing protein-coding genes ordered by their enrichment in $\mathrm{THY}^{+} / \mathrm{VEGFA}$ basal cells from human breast tumors profiled by scRNA-seq. Enrichment was defined as the number of $\mathrm{THY}^{+} / \mathrm{VEGFA}{ }^{+}$basal cells expressing a given gene (TPM $>0$ ) divided by the total number of cells expressing that gene. Only genes expressed by at least 5 basal cells were considered. LMO2 is highlighted in red. (C) Paired bar plots showing percent of $\mathrm{LMO}^{+}$cells in $\mathrm{THY}^{+} / \mathrm{VEGFA}{ }^{+}$cells (red) and all other cells (blue) in two human breast cancer datasets, including Kim et al., 2018 (4 primary triple-negative breast cancers, single nucleus RNA-sequencing, tumor only, $n=$ 659) (Kim et al., 2018), and the basal cells (see methods for details; $n=910$ ) from this study. Statistical analysis was performed by Fisher's Exact Test for association of $\mathrm{LMO}^{+}$cells with $\mathrm{THY}^{+} / \mathrm{VEGFA}{ }^{+}$cells. Individual and combined $P$ values by Fisher's method are shown in the graph. ${ }^{*} P<0.1 ;{ }^{* *} P<0.05 ;{ }^{* *} P<0.01$. (D) Heatmap depicting the top 30 differentially expressed genes, along with selected keratin and lineage markers, in $\mathrm{LMO}^{+}$( $n=7$ cells) vs. LMO2- $(n=903$ cells) basal epithelial cells from primary breast tumors. A random subsample of 50 LMO2- basal cell transcriptomes is shown for clarity. Color scale (above) represents z-score-normalized expression per gene.

(E) Differential enrichment of the 'HALLMARK_ANGIOGENESIS' pathway in $L M O 2^{+}$ vs. - in two independent human breast cancer datasets described in C. To ensure a fair comparison between $L M O 2$ positive and negative populations, an empirical $P$ value was calculated by comparing the mean enrichment in $\mathrm{LMO}^{+}$basal cells versus a sizematched collection of $L M O 2^{-}$basal cells randomly sampled 10,000 times. A combined $P$ value by Fisher's method is also shown. ${ }^{*} P<0.1 ;{ }^{* *} P<0.05 ;{ }^{* *} P<0.01$.

568 (F and G) Cell-type and survival association of $\mathrm{LMO}^{+}$basal cells across 508 bulk human breast tumor transcriptomes (Esserman et al., 2012) deconvolved using CIBERSORTx.

571 (F) Co-association patterns among cell type abundance profiles in bulk breast tumors, as quantified by Pearson correlation. Basal $\mathrm{LMO}^{+}$cells and endothelial cells are highlighted.

(G) Kaplan Meier curves showing differences in distant recurrence-free survival (DRFS) in 508 breast cancer patients stratified by the median abundance of $\mathrm{LMO}^{+}$basal epithelial cells. DRFS was modeled as a function of $\mathrm{LMO}^{+}$basal cell status and ESR1 status (Methods). The adjusted log-rank $P$ value and hazard ratio with $95 \%$ confidence interval for $\mathrm{LMO}^{+}$basal cell status is shown. 
A

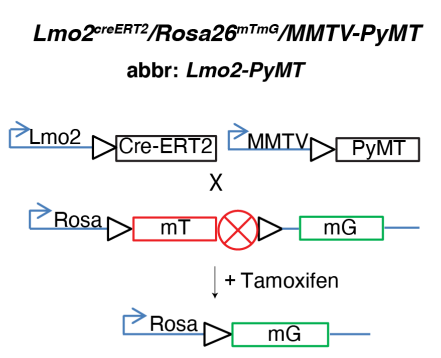

E

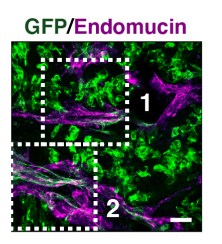

F Mammary tumor from LMO2-PyMT mice

$$
\underset{\text { Lineage deplete }}{\stackrel{\text { Dissociate to single cells }}{\longrightarrow}}
$$

G
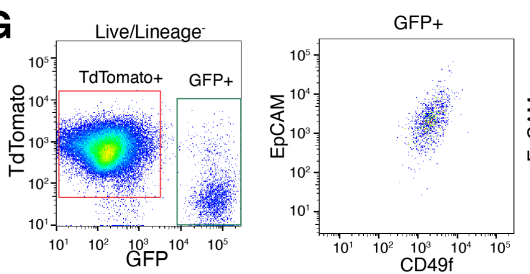
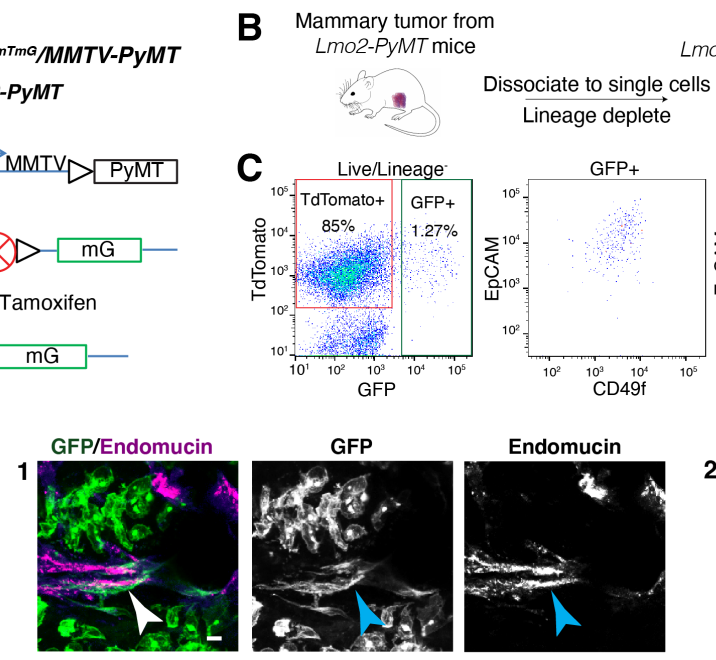

Lmo2-PyMT cells
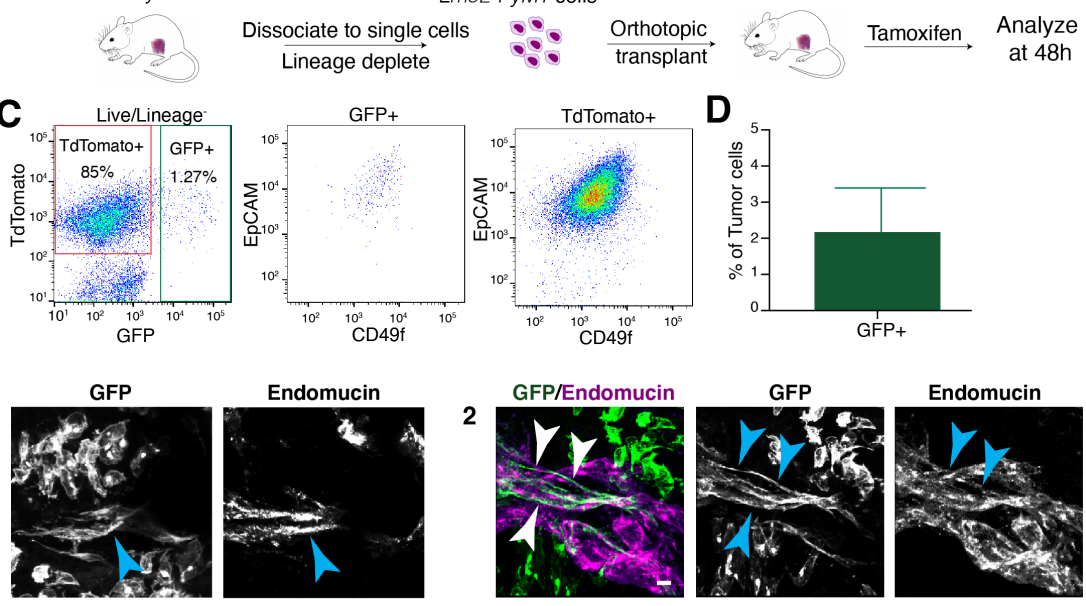

Figure 2: Lmo2 lineage-traced tumor epithelial cells integrate into the vasculature
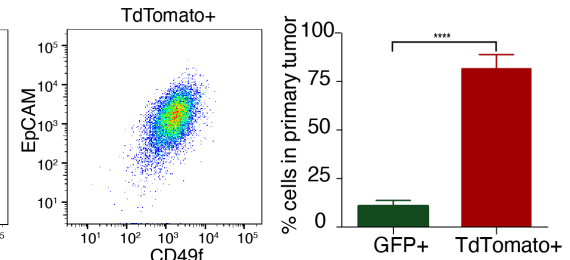

H
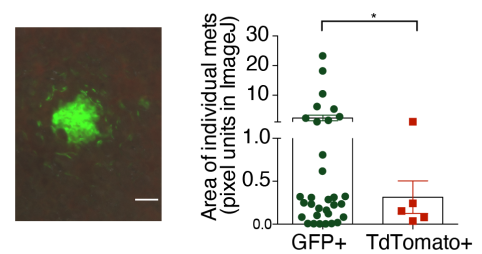
and can form metastasis in PyMT tumors.

581 (A) Schematic diagram showing generation of the triple transgenic Rosa $26^{m T m G}$ reporter with MMTV-PyMT and Lmo2-CreERT2 mice (referred to as Lmo2-PyMT).

(B) Schematic diagram showing the experimental scheme for Lmo2-PyMT tumors treated with tamoxifen.

(C) Panel 1: FACS analysis of $L m o 2-P y M T$ tumors $48 \mathrm{~h}$ after Tamoxifen pulse. Cells are gated on lineage ${ }^{-}\left(\mathrm{CD}_{4} 5^{-}, \mathrm{CD}^{-} 1^{-}\right.$, Ter119-), DAPI ${ }^{-}$cells (See Fig. S2) and analyzed using TdTomato ${ }^{+}$and $\mathrm{GFP}^{+}$. Panels 2 and 3: EpCAM and CD49f expression status in GFP $^{+}$and TdTomato ${ }^{+}$cells.

(D) Quantification of GFP ${ }^{+}$cells from Lmo2-PyMT tumors ( $n=5$ mice).

(E) Representative immunofluorescence image of $L m \circ 2$ lineage-traced cells (GFP ${ }^{+}$ green) co-localizing and integrating with endomucin (magenta) stained tumor vasculature. High resolution magnification of Inset 1 and 2 are presented, Scale bar $=$ $59350 \mu \mathrm{m}$.

594 (F) Schematic diagram showing the experimental scheme for Lmo2-PyMT tumors 595 treated with tamoxifen to trace metastatic cells. (G) Panel 1: FACS analysis of Lmo2-PyMT tumors at tumor end point from (F). Cells are gated on lineage ${ }^{-}\left(\mathrm{CD}^{-} 5^{-}, \mathrm{CD} 31^{-}, \mathrm{Ter}^{119^{-}}\right)$, DAPI ${ }^{-}$cells (See Fig. S2) and analyzed using TdTomato ${ }^{+}$and $\mathrm{GFP}^{+}$. Panels 2 and 3: EpCAM and CD49f expression status in 600 GFP $^{+}$and TdTomato ${ }^{+}$cells. Panels 4: Quantification of TdTomato ${ }^{+}$and $\mathrm{GFP}^{+}$cells from Lmo2-PyMT tumors ( $n=4$ mice). 
bioRxiv preprint doi: https://doi.org/10.1101/2021.05.26.443198; this version posted May 26, 2021. The copyright holder for this preprint (which

was not certified by peer review) is the author/funder, who has granted bioRxiv a license to display the preprint in perpetuity. It is made available under aCC-BY-NC-ND 4.0 International license.

601 (H) Panel 1: Representative image of metastasis shown, Scale bar $=100 \mu \mathrm{m}$. Panel 2:

602 Quantification of total number and area of GFP ${ }^{+}$and TdTomato ${ }^{+}$lung metastasis in

603 Lmo2-PyMT tumors. ( $n=4$ mice) Data are shown as mean $\pm \mathrm{SD}$, and statistical analysis

604 was performed by unpaired, two-sided Wilcoxon rank sum test ${ }^{*} P<0.05$. 
A

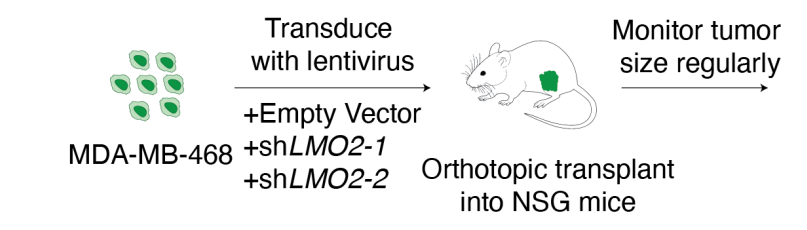

Terminate study when control tumors reach $1500-2000 \mathrm{~mm}^{3}$ into NSG mice
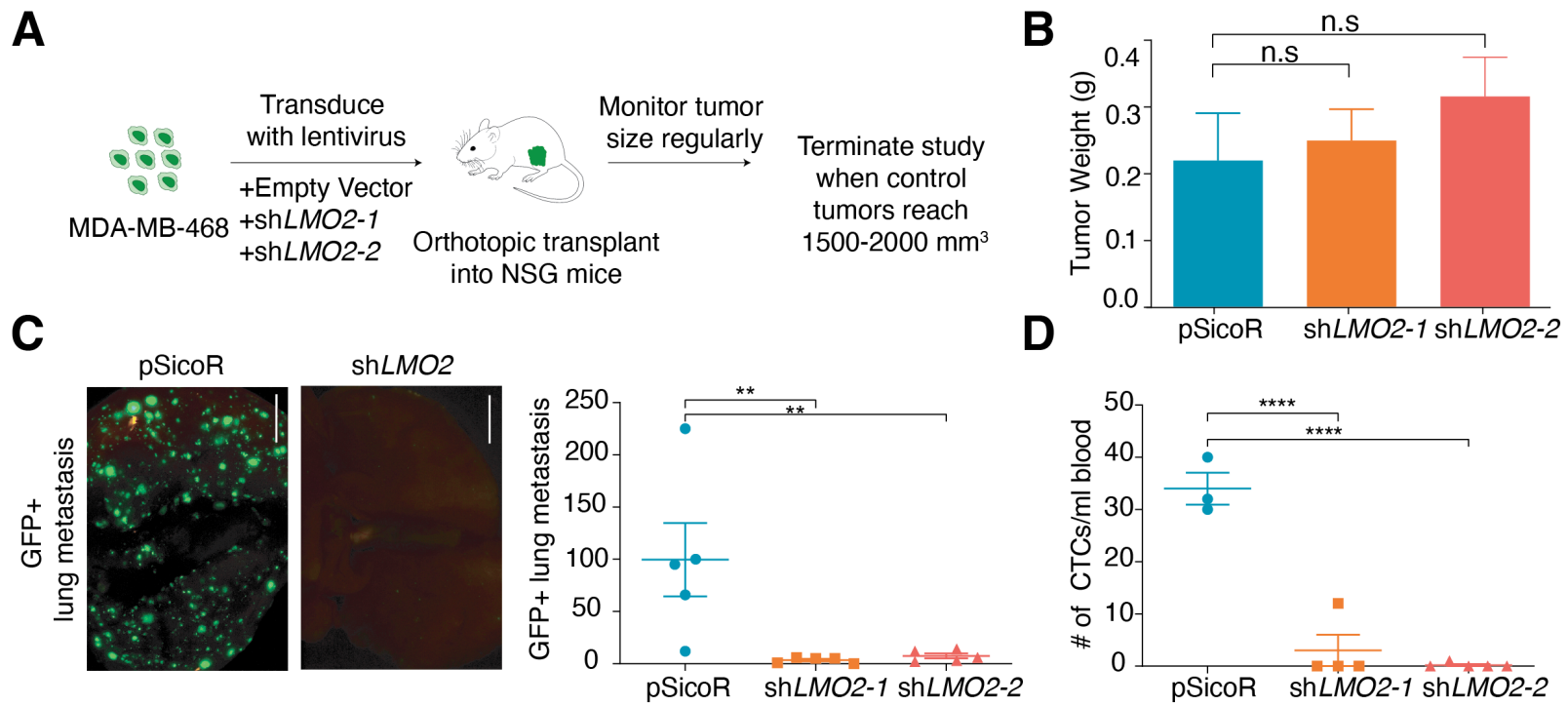

$\mathbf{E}$
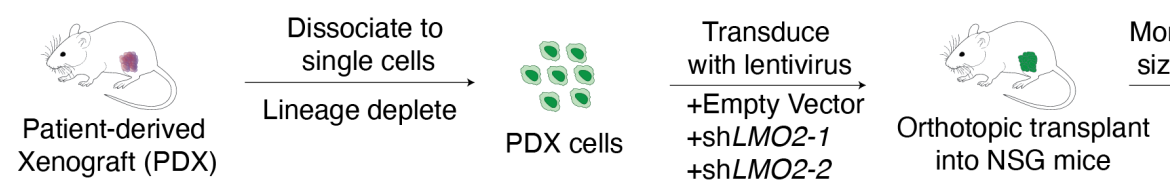

Monitor tumor

size regularly Terminate study when control tumors reach + shLMO2-2 into NSG mice

$\mathbf{F}$

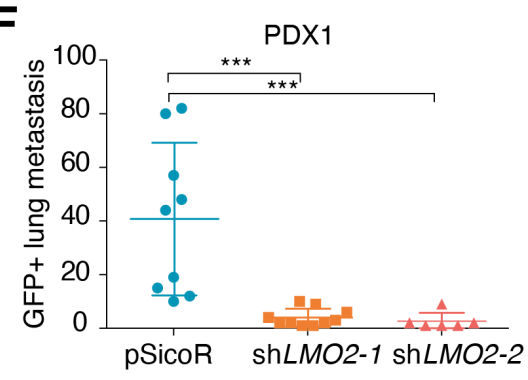

G

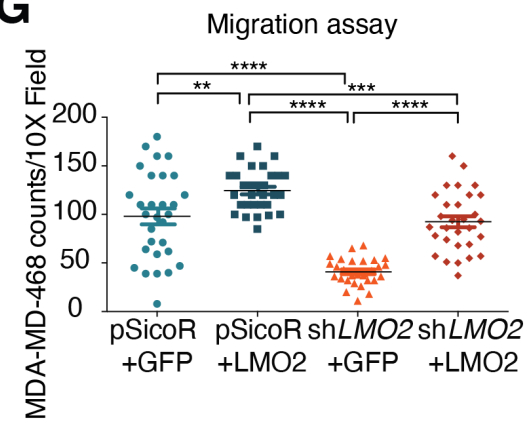

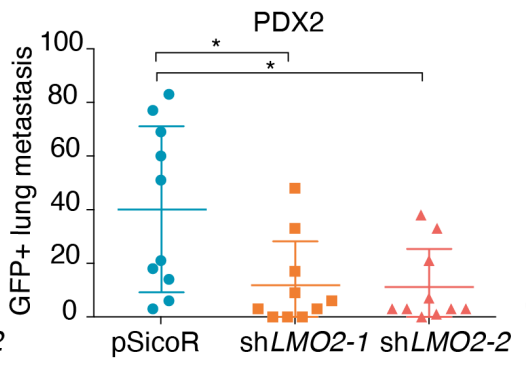

H

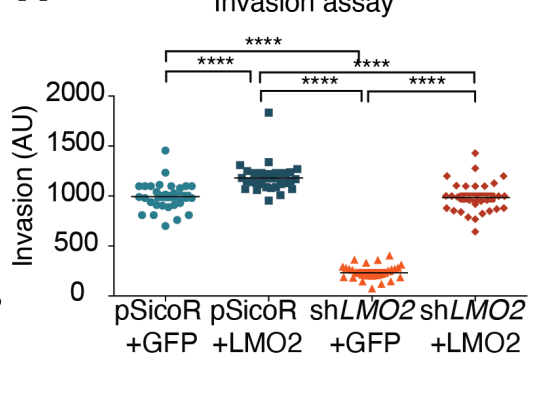

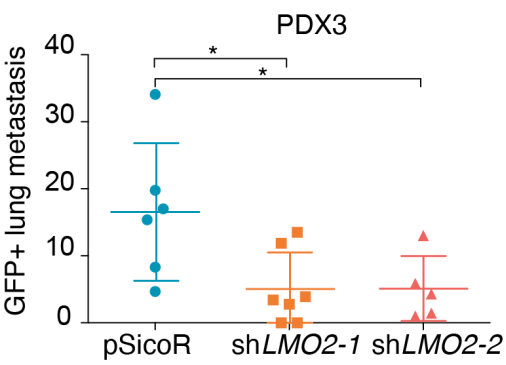

I

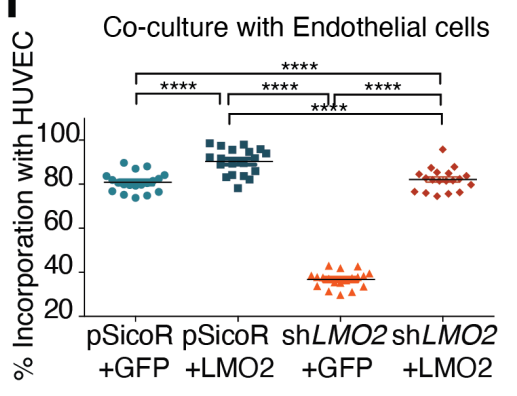

Figure 3. Knockdown of LMO2 reduces lung metastasis in human breast cancer. (A) Schematic of LMO2 knockdown in MDA-MB-468 cells followed by orthotopic transplant in NSG mice to evaluate tumor burden and metastases.

608 (B) LMO2 knockdown in MDA-MB-468 cells. Tumor weight is shown with no significant difference between the control and LMO2 knockdown ( $n=5$ mice/group). Data are shown as mean \pm SD, and statistical analysis was performed by ANOVA with Dunnett's adjustment, n.s $P>0.05$ 
612 (C) LMO2 knockdown decreases the number of spontaneous GFP ${ }^{+}$lung metastasis in 613 MDA-MB-468 cells ( $n=5 \mathrm{mice} / g r o u p)$. Left panel: representative immunofluorescence 614 image with scale bar $=5 \mathrm{~mm}$, right panel: quantification. Data are shown as mean $\pm S D$, and statistical analysis was performed by ANOVA with Dunnett's adjustment, **

$616 \quad P<0.01$.

617 (D) LMO2 knockdown decreases the number of circulating tumor cells in MDA-MB-468 618 cells ( $n=3$ mice in pSicoR, 4 in shLMO2-1, 5 in shLMO2-2). Data are shown as mean \pm 619 SD, and statistical analysis was performed by ANOVA with Dunnett's adjustment, **** $620 \quad P<0.0001$.

621 (E) Schematic of LMO2 knockdown in patient derived xenografts (PDXs) followed by 622 orthotopic transplant in NSG mice to evaluate tumor burden and metastases.

623 (F) LMO2 knockdown decreased number of spontaneous GFP ${ }^{+}$lung metastasis in PDX 624 samples. Data are combined from 3 independent experiments for PDX1, PDX3 and 625 from 2 independent experiment for PDX2 ( $n=9$ mice/ group for PDX1, $n=6$ mice/group 626 for PDX2, $n=10$ mice/group for PDX3). Data are shown as mean \pm SD, and statistical 627 analysis was performed by ANOVA with Dunnett's adjustment, ${ }^{*} P<0.05,{ }^{* *} P<0.01,{ }^{* * *}$ $628 P<0.001,{ }^{* * * *} P<0.0001$.

629 (G) MDA-MB-468 cells infected with shRNA targeting 3' UTR of LMO2 or a control shRNA pSicoR were infected with either an empty vector control 'GFP' or an LMO2overexpression vector '+LMO2' to generate pSicoR +GFP, pSicoR +LMO2, shLMO2 632 +GFP, shLMO2 +LMO2. Transwell migration was quantification at 24 hours.

633 (H) Spheroid invasion assay was performed and quantified at Day 5 using the breast 634 cancer cells from $(\mathbf{G})$.

635 (I) The breast cancer cells from (G) were co-cultured with HUVEC cells and the 636 percentage of breast cancer cells that are co-localizing with HUVEC tubes was 637 quantified using ImageJ.

638 For all experiments in (G-I), $n=3$ and 10 images were analyzed per condition per $n$. Statistical analysis was performed by ANOVA with Dunnett's adjustment, and significance is indicated as ${ }^{* *} P<0.01,{ }^{* * *} P<0.001,{ }^{* * *} P<0.0001$ 
A
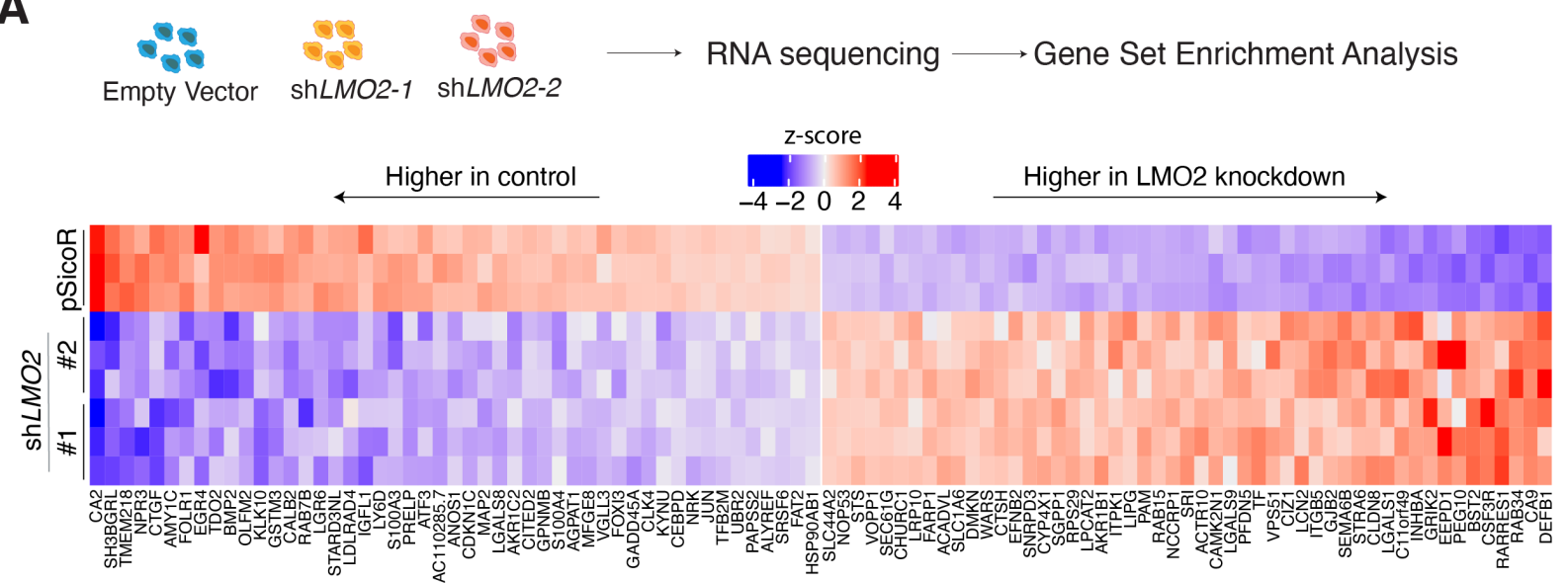

B

\begin{tabular}{|c|c|c|}
\hline Pathway & NES & FDR Q-value \\
\hline TNFa Signaling Via NFKB & -3.89 & 0.00 \\
\hline E2F Targets & -1.97 & 0.05 \\
\hline IL6-JAK-STAT3 Signaling & -1.89 & 0.06 \\
\hline Interferon Gamma Response & -1.85 & 0.06 \\
\hline Apoptosis & -1.80 & 0.05 \\
\hline Hypoxia & -1.80 & 0.05 \\
\hline KRAS Signaling UP & -1.75 & 0.06 \\
\hline
\end{tabular}

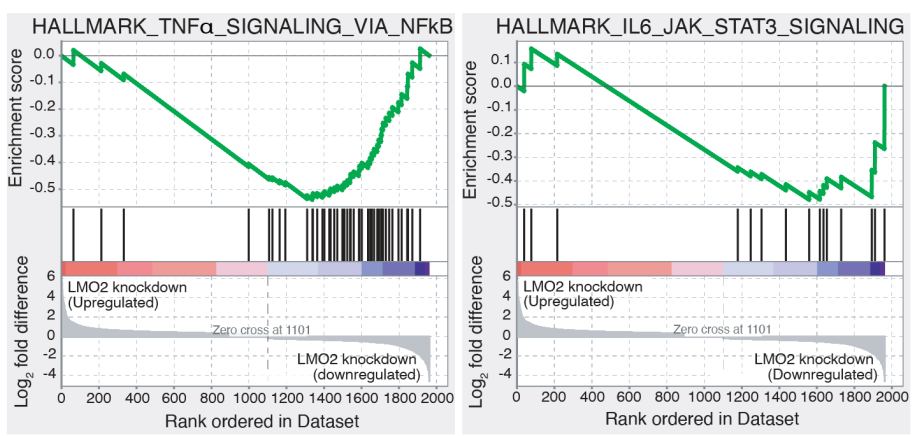

C

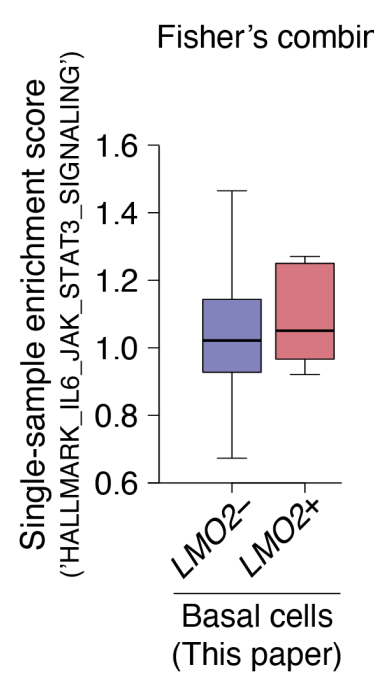

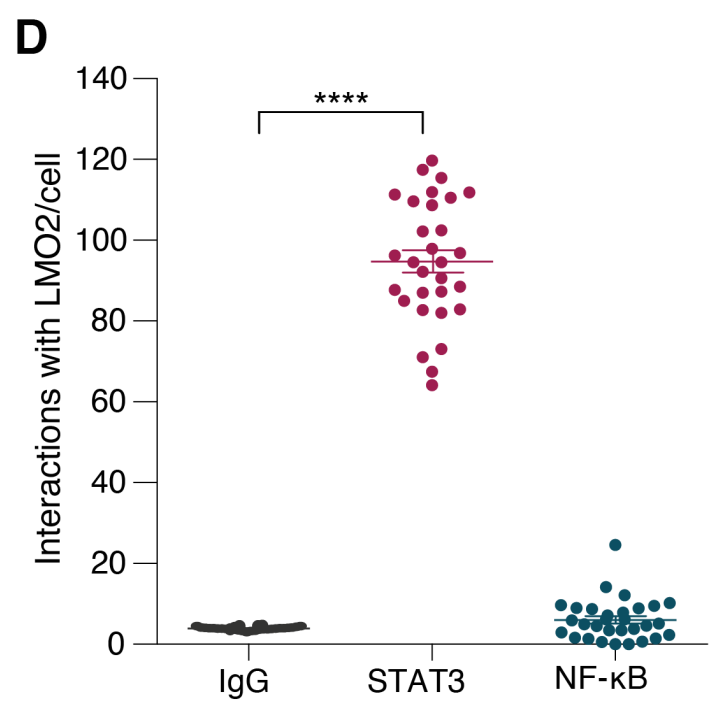

641 Figure 4: LMO2 regulates the IL6-JAK-STAT3 pathway and binds to STAT3

642 (A) Top: Schematic of bulk RNA-sequencing analysis in MDA-MB-468 cells infected 643 with shRNAs targeting LMO2 or a control pSicoR. Bottom: Heatmap showing top and 644 bottom 50 genes differentially expressed between control and LMO2 knockdown 645 conditions, ordered by $P$-adjusted value. 
646 (B) Left: Hallmark gene sets found to be significantly enriched by GSEA analysis.

647 Normalized enrichment scores (corresponding to control pSicoR vs LMO2 knockdown)

648 and FDR $Q$-values are determined by the GSEA software. An FDR $Q$-value cutoff of

$649<0.25$ was used to select significant gene sets. Right: Enrichment plots for

650 'HALLMARK_TNF $\alpha$ SIGNALING_VIA

651 _NFkB' and 'HALLMARK_IL6_JAK_STAT3_SIGNALING' are depicted.

652 (C) Differential enrichment of the 'HALLMARK_IL6_JAK_STAT3_SIGNALING' pathway

653 in $\mathrm{LMO}^{+}$vs. ${ }^{-}$cells from two independent human breast cancer datasets as described

654 in Fig. 1C.

655 (D) Proximity mediated ligation assay showed that LMO2 had a stronger interaction with 656 STAT3 compared to NF-kB in vitro ( $n=3,10$ images were analyzed per condition per $n$ ).

657 Statistical analysis was performed by ANOVA with Dunnett's adjustment. ${ }^{* * *} P<0.0001$ 
A
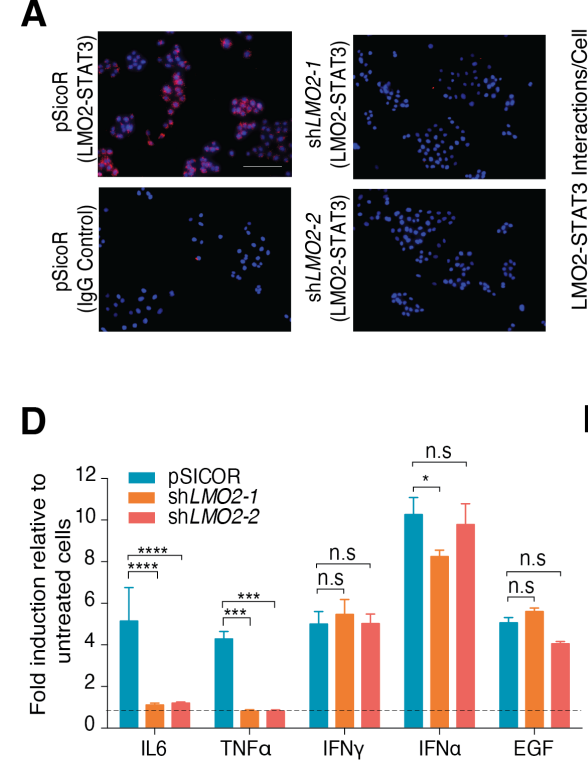

F

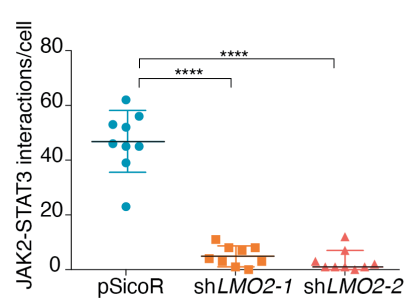

G

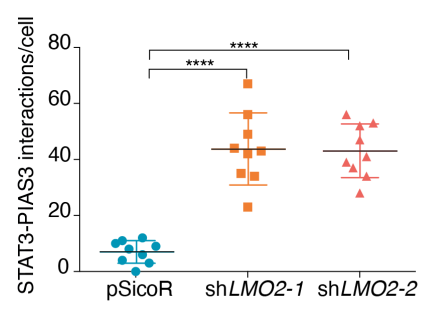

B
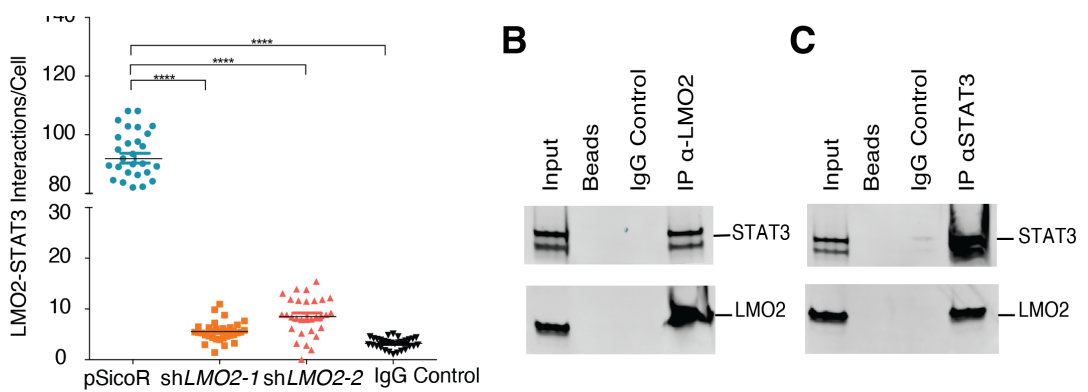

E
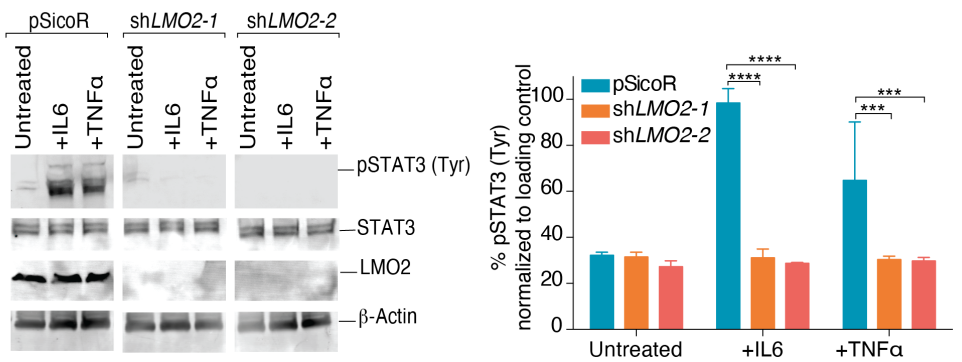

H

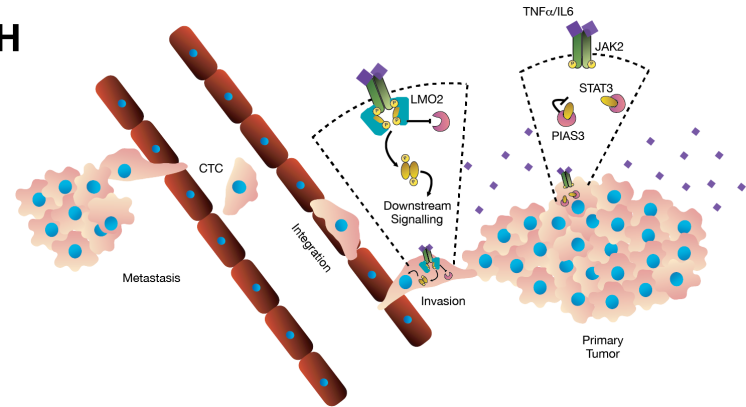

Figure 5: LMO2 stabilizes STAT3 signaling in breast cancer cells

(A) Left panel: Proximity mediated ligation assay shows that LMO2 binds to STAT3 in vitro and this interaction is significantly reduced with LMO2 knockdown indicating specificity of the assay. Right panel: Quantification of $n=3$ experiments and 10 images were analyzed per condition per $n$. Scale bar $=60 \mu \mathrm{m}$. Statistical analysis was performed by ANOVA with Dunnett's adjustment, ${ }^{* * *} P<0.0001$. LMO2 shows that LMO2 is able to pull-down STAT3. One representative blot of three independent experiments in shown. (C) Western blot of the input, immunoprecipitated beads (control), IgG (control) and STAT3 shows that STAT3 is able to pull-down LMO2. One representative blot of three independent experiments in shown.

(D) STAT3-luciferase reporter activity shows robust stimulation of luciferase in control but not in cells with LMO2 knockdown when treated with IL6 and TNF $\alpha$. IFN $\alpha$, IFN $\gamma$, EGF treatment of cells results in robust stimulation in control and knockdown cells suggesting that LMO2 function is specific to IL6 and TNF $\alpha$. Quantification of $n=3$ experiments. Statistical analysis was performed by 2-way ANOVA with Sidak's correction, and significance is indicated as ${ }^{* *} P<0.01,{ }^{* * *} P<0.001$, n.s. $P>0.05$. 
678 and TNF $\alpha$ indicating that LMO2 knockdown disrupts phosphorylation of STAT3.

679 Quantification of $n=3$ experiments. Statistical analysis was performed by 2-way ANOVA 680 with Sidak's correction, and significance is indicated as ${ }^{* *} P<0.001$, ${ }^{* * * *} P<0.0001$. 681 (F) Interactions between STAT3 and JAK2 detected by proximity mediated ligation 682 assay is reduced upon LMO2 knockdown indicating that LMO2 facilitates binding of 683 STAT3 and JAK2. Quantification of $n=3$ experiments. Statistical analysis was performed 684 by ANOVA with Dunnett's adjustment, and significance is indicated as ${ }^{* * * *} P<0.0001$. 685 (G) Interactions between STAT3 and PIAS3 detected by Proximity mediated ligation 686 assay is increased upon $\mathrm{LMO} 2$ knockdown indicating that LMO2 prevents binding of 687 STAT3 and PIAS3. Quantification of $n=3$ experiments. Statistical analysis was 688 performed by ANOVA with Dunnett's adjustment, and significance is indicated as **** $689 \quad P<0.0001$.

690 (H) Schematic of proposed mechanism of LMO2 in breast cancer metastasis. Tumor 691 cells that express LMO2 have stabilized STAT3 signaling in response to IL6 and TNF $\alpha$ 692 from the microenvironment, allowing these cells to intravasate into the circulation by 693 incorporating into the vasculature. 